\title{
Individual prediction of thrombocytopenia at next chemotherapy cycle - a model comparison
}

\author{
Yuri Kheifetz ${ }^{1}$ and Markus Scholz ${ }^{1}$ \\ ${ }^{1}$ Leipzig University
}

June 10, 2020

\begin{abstract}
Aims: Thrombocytopoenia is a common major side-effect of cytotoxic cancer therapies. A clinically relevant problem is to predict an individual's thrombotoxicity in the next planned chemotherapy cycle in order to decide on treatment adaptation. To support this task, two dynamical mathematical models of thrombopoiesis under chemotherapy were proposed, a simple semi-mechanistic model and a comprehensive mechanistic model. In this study, we compare the performance of these models. Methods: We consider close-meshed individual time series data of 135 non-Hodgkin's lymphoma patients treated with six cycles of CHOP/CHOEP chemotherapies. Individual parameter estimates were derived on the basis of these data considering a varying number of cycles per patient. Parsimony assumptions were applied to optimize parameter identifiability. Models are compared by determining deviations of predicted and observed degrees of thrombocytopoenia in the next cycles. Results: The mechanistic model results in superior fits of individual time series data. Moreover, prediction accuracy of future cycle toxicities by the mechanistic model is higher even if it used data of two cycles, while the semi-mechanistic model used data of five cycles for the corresponding calibrations. Conclusions: We successfully established a quantitative and clinically relevant method for comparing prediction performance of biomathematical models of thrombopoiesis under chemotherapy. We showed that the more comprehensive mechanistic model outperforms the semi-mechanistic model. We aim at implementing the mechanistic model into clinical practice to assess its utility in real life clinical decision making
\end{abstract}

\section{Individual prediction of thrombocytopenia at next chemotherapy cycle - a model comparison}

Short running title:

\section{Individualized prediction of thrombocytopenia during chemotherapy}

Yuri Kheifetz, Markus Scholz

Institute for Medical Informatics, Statistics and Epidemiology, University of Leipzig, Haertelstrasse 16-18, 04107 Leipzig, Germany

\section{Corresponding author:}

Markus Scholz: : markus.scholz@imise.uni-leipzig.de

Key Words:

Pharmacometrics, Pharmacodynamics, Adverse Drug Reactions, Medical

Oncology (Oncology), platelets (Haematology) 


\section{Principle investigator:}

The notion of "principle investigator" is not applicable in our case. We performed no studies with human subjects/patients. All data are derived from published clinical studies.

Word count: 4000 words.

Table count: No tables in the main text.

Figures count: 8 figures.

Statement 1 : What is already known about this subject:

- Severity of haematotoxicity under chemotherapy is heterogeneous among patients and difficult to predict

- Biomathematical models of thrombopoiesis were developed and showed potential to describe individual therapy courses

- A tool for neutrophil-guided dose adaptation in chemotherapy based on a semi-mechanistic haematopoiesis model is available

Statement 2 : What this study adds:

- We established a novel quantitative and clinically relevant method for comparing prediction performance of different biomathematical models of thrombopoiesis under chemotherapy.

- We applied this method to compare the individual prediction performance of a comprehensive mechanistic and a semi-mechanistic model regarding thrombocytopenia of grades III-IV.

- We analysed the contribution of baseline covariates to prediction performance of the models using an independent validation data set

- We showed that the comprehensive mechanistic model has superior predictive accuracy compared to the semi-mechanistic model

\section{Summary}

Aims: Thrombocytopoenia is a common major side-effect of cytotoxic cancer therapies. A clinically relevant problem is to predict an individual's thrombotoxicity in the next planned chemotherapy cycle in order to decide on treatment adaptation. To support this task, two dynamical mathematical models of thrombopoiesis under chemotherapy were proposed, a simple semi-mechanistic model and a comprehensive mechanistic model. In this study, we compare the performance of these models.

Methods: We consider close-meshed individual time series data of 135 non-Hodgkin's lymphoma patients treated with six cycles of $\mathrm{CHOP} / \mathrm{CHOEP}$ chemotherapies. Individual parameter estimates were derived on the basis of these data considering a varying number of cycles per patient. Parsimony assumptions were applied to optimize parameter identifiability. Models are compared by determining deviations of predicted and observed degrees of thrombocytopoenia in the next cycles.

Results: The mechanistic model results in superior fits of individual time series data. Moreover, prediction accuracy of future cycle toxicities by the mechanistic model is higher even if it used data of two cycles, while the semi-mechanistic model used data of five cycles for the corresponding calibrations.

Conclusions: We successfully established a quantitative and clinically relevant method for comparing prediction performance of biomathematical models of thrombopoiesis under chemotherapy. We showed that the more comprehensive mechanistic model outperforms the semi-mechanistic model. We aim at implementing the mechanistic model into clinical practice to assess its utility in real life clinical decision making. 


\section{Introduction}

Thrombocytopoenia is a major side effect of many anti-cancer cytotoxic drugs and is dose-limiting in some occasions. However, severity of this condition is highly heterogeneous between patients with the effect that a possibly small proportion of patients expressing excessive toxicity limits the dosage for the entire patient population for safety reasons. It is therefore of clinical importance to predict thrombotoxicity on an individual level and to develop and apply personalized schedules of chemotherapy and ameliorative treatment such as platelet transfusion or growth factor applications.

A practically applied method to personalize chemotherapy are dose adjustments which could be done either a priori or a posteriori. A priori dose adjustment procedures are based on pharmacogenetic, demographic and clinical information with known predictive power regarding cytotoxic effects. However, although several of such risk factors are known, their predictive power is poor, still resulting in high heterogeneity within risk groups. Since current chemotherapy regimens are based on multiple cycles, aposteriori dose adjustment referring to therapy control on the basis of observed toxicity could be a promising alternative. A posteriori Bayesian-guided dosing for several anticancer drugs was applied by different groups as reviewed in Rousseau et al . Based on a semi-mechanistic model of haematopoiesis developed by Friberg et al. , a Bayesian $a$ posteriori strategy of neutrophil-guided dose adaptation was proposed and a predictive tool was developed . However, it was shown, that inter-occasion variability (IOV) reduced effectiveness of dose adaptations. The authors treated IOV as a purely random effect affecting some of the individualized parameters. However, IOV of haematotoxicity could result from (non-random) long-range cumulative chemotherapy effects. To account for this effect, modifications of the Friberg model were proposed . Among these, the model of Henrich et al was designed to consider cumulative chemotherapy-induced haematotoxicity, based on slow bone-marrow exhaustion .

We recently proposed a comprehensive model of human thrombopoiesis under chemotherapy and developed a method to fit it to individual patient data using a Bayesian approach which exploits a large body of literature information including patient data of other studies. The major aim of the present work is to propose a framework to assess, improve and compare the performance of different models regarding prediction of nextcycle thrombocytopoenia at an individual level. We apply this framework to compare our model with that of Henrich et al .

\section{Materials and methods}

\section{Modelling concepts to be compared}

We compared the predictive potential of our comprehensive mechanistic thrombopoiesis model with the semi-mechanistic model of general haematopoiesis and bone marrow exhaustion proposed by Henrich et al . Figure 1 provides and overview of both modelling concepts.

\section{Figure 11.}

We present the underlying modelling concepts in brief in the following.

Semi-mechanistic haematopoiesis model

The semi-mechanistic ODE model of Henrich at al (Figure 1A) is proposed as improvement of the Friberg model. Compared to this model, it adds a slowly replicating pluripotent stem cell compartment (Stem ) at the beginning of the compartment chain intended to represent general bone marrow proliferation and maturation. This chain includes a proliferation compartment (Prol ), three equal transit compartments mirroring maturation of blasts (Transit) and one circulating compartment (Circ). Proliferation in Stem and Prol are controlled by proliferation rate constants $k_{s t e m}$ and $k_{\text {prol }}$, respectively. For the sake of parsimony, the same parameter $k_{t r}$ describes transition rates between the five sequential compartments starting from Prol , the rate of cell division under steady state condition at the proliferation compartments and a degradation rate of circulating cells. 
A feedback loop from relative counts of circulating cells is imposed on both, Stem and Prol compartment. More precisely, the term $\left(\frac{\operatorname{Circ}_{0}}{\operatorname{Circ}}\right)^{\gamma}$ serves as a feedback factor of the proliferation, where Circ $_{0}$ is the steadystate value of Circand $\gamma$ is a sensitivity parameter. As a consequence, more circulating cells imply reduced proliferation. Chemotherapy toxicity is modelled as a factor reducing proliferation in Stem and Prol (see Supporting information S.2 for details).

We borrowed PK models of etoposide, cyclophosphamide and doxorubicin from other authors and attached it to that model. One pharmacodynamical (PD) effect per cytotoxic drug is assumed for the proliferation compartments (Stem and Prol ) only. Relations between PD effects of different drugs are derived from other studies. We assumed inter-individual variability (IIV) for four parameters of this semi-mechanistic model based on parsimony analyses (see Table S.4.4 and explanations provided in the Supporting information S.4).

Comprehensive mechanistic thrombocytopoiesis model

Our mechanistic model is an updated and individualized version of our previous work. Model structure as well as all biological assumptions, mathematical equations and parameter estimation can be found elsewhere - In brief, the model describes the dynamics of active and dormant stem cells, colony-forming units of megakaryocytes, megakaryocytes, of different ploidies and platelets in both spleen and circulation. The model contains the following three feedback loops (see also Figure 1B and Supporting information S.1):

1. Autoregulation feedback loop: Self-renewal of stem cells is negatively regulated by the relative count of active stem cells.

2. Intermediate range feedback loop: There is a negative feedback loop of higher megakaryocyte numbers increasing the transition of active stem and CM cells to the respective dormant states effectively blocking further stem cell differentiations into thrombocytopoiesis lineage in case of sufficiently high numbers of megakaryocytes.

3. Feedback loop of blood to bone marrow. This feedback is mediated by the growth factor thrombopoietin (TPO).

4. TPO is produced in the liver and the kidneys at constant rate.

5. TPO activates dormant stem and progenitor cells in a delayed way, increases the ploidy of megakaryocytes, activates dormant megakaryocytes and suppresses platelet formation.

6. TPO is actively consumed by megakaryocytes and platelets in blood. It is also cleared via the kidneys.

An important novelty of our model compared to other mechanistic models of thrombopoiesis is the incorporation of a model of bone-remodeling developed by Komarova et al. . This model describes the interaction of bone-supporting osteoblasts and bone-destructing osteoclasts. The model is linked to our model of thrombocytopoiesis by assuming that osteoblasts are required to maintain dormant cells of stem cell, CM and megakaryocyte compartments. In more detail, we assume that osteoblasts define the capacity of bone marrow, i.e. higher osteoblast count implies a higher capacity for dormant cells of all types. This is supported by studies showing an involvement of megakaryocytes and osteoblasts in supporting stem cells niches .

The effect of cytotoxic drugs on bone marrow is modelled by a depletion of proliferating stem cells, progenitor cells, megakaryocytes, osteoblasts and osteoclasts. Elimination of osteoblasts through multi-cyclic chemotherapy results in a long-term reduction of the bone marrow capacity, and with it, reduction of dormant stem cells and megakaryocytes. This assumption enables us for the first time to mechanistically describe cumulative long-term toxicity as observed in multi-cycle chemotherapy. We used the same PK models of etoposide, cyclophosphamide and doxorubicin and the same relations between PD effects of different drugs as described for the semi-mechanistic model.

To calibrated our mechanistic model, we developed and applied a novel approach of integration of several clinical data sets, the so-called principle of virtual participation . In brief, we assumed that single patients also did participate in other studies and penalize large deviations from these data. Details are described in the Supporting information S.4. As a consequence, the model is required to be consistent with several other data resources which greatly stabilizes individual parameter estimates even if the individual data are relatively sparse. As a result of parsimony assumptions, a total of 12 parameters of the mechanistic model 
are assumed to show IIV (see Table S.2 of the Supporting information). Without applying the principle of virtual participation, the number of identifiable parameters would drop to four. Goodness of fit would also drop considerably (not shown).

We implemented and simulated the semi-mechanistic model in Matlab, using 15s ODE (ordinary differential equations) solver . Implementation and simulations of our mechanistic model is performed in $\mathrm{R}$ and $\mathrm{C}++$, using package Rcpp. ODE equations have been approximated by the Bogacki-Shampine method .

\section{Comparison of the predictive potential of the models regarding next cycle toxicity}

Here we describe, how the models are compared regarding their predictive potential of individual next cycle toxicity. The general idea is that the models are fitted on platelet time series of patients observed in a prescribed number of cycles. Based on the resulting individual parameter estimates, we then simulated the next cycle for that patient, i.e. we predicted the thrombotoxicity for the next cycle. Individual treatment adaptations are considered throughout these processes by simulating the therapy actually applied to that patient.

Simulation results of both models are compared with observed platelet counts using a negative log-likelihood function (see section S.4 in the Supporting information). To assess the relative advantages of the models, we also performed comparisons of model predictions based on differing numbers of cycles used for parameter fitting. Quality of agreement of observed and simulated platelet counts was mainly assessed on the basis of the smallest observed cell counts because these values are typically used to define the degree of toxicity even though smaller values are possible when there is no measurement in the true nadir phase of platelets. We are confined to the following WHO degrees of toxicity for that purpose:

- Degree 0, nadir $>150 \cdot 10^{9}$ cells $/$ L.

- Degree 1 , nadir between $75 \cdot 10^{9}$ and $150 \cdot 10^{9}$ cells/L.

- Degree 2, nadir between $50 \cdot 10^{9}$ and $75 \cdot 10^{9}$ cells/L.

- Degree 3, nadir between $25 \cdot 10^{9}$ and $50 \cdot 10^{9}$ cells/L.

- Degree 4 , nadir $<25 \cdot 10^{9}$ cells/L.

To evaluate the prediction quality of a model, we determined the average of the absolute differences between individually observed and predicted degrees of thrombocytopenia (also called difference of degrees (DD) in the following)

$$
\begin{aligned}
& \mathrm{DD}_{r, k}=\frac{\sum_{i=1}^{L_{k}}\left|\mathrm{SD}_{i, r, k}-\mathrm{OD}_{i, r, k}\right|}{L_{k}} \\
& \quad, \quad a m p ; r=1, \cdots, N_{i}-1, \quad a m p ; k=r+1, \cdots, N_{i},(1)
\end{aligned}
$$

where $r$ is the number of cycles used for calibration, $k$ is the cycle for which the predictions are made, and $L_{k}$ denote the number of patients analyzed in cycle $k . S D_{i, r, k}$ and $O D_{i, r, k}$ correspond respectively to the simulated (predicted) and observed degrees of thrombocytopenia of patient $i$ in cycle $k$. The reciprocal of this error term served as an operationalization of the predictive power of the model.

As an alternative measure of prediction quality, we only counted larger differences between observed and predicted degree of thrombocytopenia, namely differences of more than one degree. Again, we averaged these differences over the number of patients for which a prediction is made (Large difference of degrees, LDD):

$\mathrm{LDD}_{r, k}=\frac{\sum_{i=1,\left|\mathrm{SD}_{i, k}-\mathrm{OD}_{i, k}\right|>1}^{L_{k}}\left|\mathrm{SD}_{i, k}-\mathrm{OD}_{i, k}\right|}{L_{k}}$

To compare the semi-mechanistic and the mechanistic model, we introduced specific notations for the outcome measures of interest, namely $\left\{\mathrm{SDD}_{\mathrm{r}, \mathrm{k}},\left\{\mathrm{SLDD}_{\mathrm{r}, \mathrm{k}}\right\},\left\{\mathrm{MDD}_{\mathrm{r}, \mathrm{k}}\right\}\right.$ and $\left\{\mathrm{MLDD}_{\mathrm{r}, \mathrm{k}}\right\}$. Here prefix $\mathrm{S}$ corresponds to the semi-mechanistic model, while prefix $\mathrm{M}$ corresponds to the statistics of the mechanistic model.

Due to clinical importance, we also compared receiver operating characteristics for the prediction of severe grade 3-4 thrombocytopenia for both models. 


\section{Clinical data}

Primary data set

Our model comparison is based on clinical data of the NHL-B trial of elderly patients treated for aggressive non-Hodgkin's lymphoma. Patients are randomized to one of the four arms 6xCHOP or 6xCHOEP with either 14 or 21 days of cycle duration. Schedules with 14 day cycle duration are supported by G-CSF at cycle days 4-13. Thrombocytopenia was treated with platelet transfusions, postponement of therapy or reduction in chemotherapy dose by $25 \%$ or $50 \%$.

We selected a total of 135 patients whose platelets counts are measured during four or more cycles with at least five measurements per cycle to obtain sufficiently detailed individual time series data. Distributions of thrombocytopenia degrees of these patients in each treatment cycle is shown in Figure 2. Average degree of thrombocytopenia increases from 0.74 at cycle 1 to 1.53 at cycle 6 . The fraction of severe thrombocytopenia (degrees 3-4) increases from $3 \%$ at cycle 1 to $24.3 \%$ at cycle 6 . For 16 patients, the last one or two cycles could not been applied due to excessive toxicity. Nine of these had thrombocytopenia of degrees 3-4 prior to therapy withdrawal.

Validation set

Since only $8.5 \%$ of the $\sim 1600$ NHL patients met our inclusion criteria, we estimated individual parameters of additional 143 NHL patients (validation set) fulfilling relaxed inclusion criteria (monitoring during four or more cycles with at least four measurements per cycle) in order to compare their distributions with those obtained for the primary data set. In this data set, platelet transfusions were not excluded. Thus, it is necessary to account for this additional treatment by assuming an efficacy parameter of platelet transfusions, which was estimated on the basis of full time series.

\section{Relationship of model parameters to clinical risk factors}

We analysed the relationship between clinical risk factors (age, sex, weight, height, body surface area, blood volume, WHO infection grade and LDH) and the derived individual model parameters by formal regression analysis. Individual parameter estimates resulting from fitting all cycles of the validation data set were used for that purpose. Details of regression analysis are explained in the Supplement Material S.5. To check the performance of these regression models, we applied them to our primary data set adding a new prediction scenario, which was only based on the clinical risk factors of the patient.

\section{Figure 21.}

\section{Results}

\section{Results of individual parameter fitting}

In order to demonstrate the capabilities of the models, we first fitted them to complete individual time series data of the 135 patients of the primary data set. Resulting distributions of individual parameter settings are presented in Supporting information S.4, Supporting Tables S.4.1 for the mechanistic model. Both models showed good agreements with data in cases where the platelet nadirs of cycles are continuously declining during the course of therapy as shown in Figure 3. Of note, the semi-mechanistic model fits the first cycle better, while the mechanistic model fits the later cycles better. In cases of irregular dynamics without clear nadir trends, both models show stronger deviations from the data of up to 1-2 grades of thrombocytopenia as shown in Figure 4. For most patients with such irregular behavior, the mechanistic model outperforms the semi-mechanistic model (patients 14, 15, 398 and 1017). Irregular time courses are frequent especially for 14-day schedules which are supported by G-CSF administrations.

\section{Figure 31.}

\section{Figure 41 .}




\section{Comparison of prediction performance}

We then fitted the model to a reduced number of cycles (1-5) and predicted the respective next cycle toxicity. We also used the individual parameters estimated by the regression models of clinical risk factors to predict toxicity of cycles without utilizing any platelet data under therapy. Results of our outcome measures $\left\{\mathrm{MDD}_{\mathrm{r}, \mathrm{k}}\right\}$ and $\left\{\mathrm{SDD}_{\mathrm{r}, \mathrm{k}}\right\} 1[?] \mathrm{r}<\mathrm{k}[?] 6$ for the mechanistic and semi-mechanistic models are displayed in Figure 5. The mechanistic model shows clearly better predictive power than the semi-mechanistic model for predicting cycles 2-6. The predictive performance of the parameters obtained from regression analysis of clinical factors are comparable for the two models. Prediction performance of the semi-mechanistic model improves only slightly when adding the cycle data. In contrast, the prediction performance of the mechanistic model clearly improves when adding cycle information, especially after adding at least one cycle. The same applies when considering LDD rather than the DD as primary measure of prediction performance (see Figure $6)$.

\section{Figure 5l.}

Of note, the mechanistic model is still more reliable when the semi-mechanistic model is calibrated on more cycles. For example, the mechanistic model fitted on the first cycle results in better prediction of toxicities in cycles 4-6 than the SMM calibrated on all cycles prior to the predicted one (see Figure 5). The superiority of the mechanistic model is considerably larger when considering LDD statistics rather than DD statistics (see Figure 6). Only the parameter values derived from the regression of clinical factors resulted in comparable prediction performance of the two models. All scenarios using cycle data resulted in superior performance of the mechanistic model except for the highly unbalanced situation where the SMM was calibrated on five cycles while the mechanistic model was calibrated on only one cycle to predict the toxicity of the last cycle.

\section{Figure 61.}

In order to check, whether the selection of patients used for the primary data set impose a bias on the resulting parameter distribution, we fitted both models to the sparser data of additional 146 NHL patients fulfilling weaker inclusion criteria. Table S.1.2 in Supporting Information S.1 provides resulting individual parameters distributions. Results are compared with the corresponding distributions of the primary data set using Welch's t-test (unequal sample size, unequal variances). No significant differences were detected for the parameters of both models indicating that bias caused by patient selection is small.

We analysed whether clinical risk factors are related to our individual parameter estimates derived from fitting all cycles (Supporting information S.5). Several significant associations were found. The pharmacodynamics effect of cyclophosphamide showed the strongest dependence on clinical factors. Most of these dependences are consistently observed for both data sets.

We finally determined sensitivity as well as the Youden index of both models to predict severe grade 3-4 thrombopoenia at the next cycle. The mechanistic model showed superior sensitivity (Figure 7) prediction performance throughout (Figure 8).

\section{Figure 7l.}

\section{Figure 81.}

\section{Discussion}

Haematotoxic side effects of cytotoxic chemotherapy are common but its severity is highly heterogeneous across patients. Intelligent strategies for individualized treatment decisions are required to optimize anticancer effect but limit toxicity to an acceptable level at the same time. However, predicting haematotoxic side effects on an individual level is a difficult task due to the underlying complex non-linear interactions of damaging and stimulating effects during cytotoxic drug treatment accompanied by a rapid turn-over of blood cells. Thus, sophisticated prediction models are required to capture essential biological features of these processes. 
In the present paper, we compared the predictive potential of two dynamical models of thrombocytopoiesis under chemotherapy proposed in the literature, a semi-mechanistic model developed for general hematopoiesis and a more comprehensive model specifically developed for thrombocytopoiesis developed by us which also considers mechanisms of the bone marrow niche interaction .

Until recently, Friberg's semi-mechanistic model was the only one applied to individual patient's data to a larger extent . Based on a Bayesian a posteriori- approach, it was used for example to develop neutrophilguided dose adaptations for etoposide and a respective computer tool was proposed . Sandström et al and Wallin et al treated IOV as a random effect affecting individual parameters which could not explain accumulating haematotoxicity. To improve this situation, Henrich et al recently introduced a slow bonemarrow exhaustion to the Friberg model. However other long range effects such as TPO activation of stem cells observed in animals as well as long-range effects on human megakaryocytes can make platelets dynamics much more complex and individually diverse implying necessity of more refined individualized mechanistic models of haematopoiesis.

Indeed, we found that our mechanistic model clearly outperforms the semi-mechanistic model regarding prediction of individual cycle toxicities especially in case of irregular platelet responses which are common for more intense chemotherapy regimens supported by growth-factor applications such as G-CSF. Combination of slow bone-marrow exhaustion with delayed TPO effects can result in a range of complex dynamical patterns, especially for short treatment cycles insufficient to fully replenish haematopoiesis. Our mechanistic model of bone marrow exhaustion is based on the observation that osteoblasts are depleted during multi-cycle chemotherapy . This aspect contributes to the better predictive power of the mechanistic model.

To fit this comprehensive model on individual level data, we applied the principle of "virtual participation", which is superior to standard mixed-model analyses since it allows exploiting external data sets to reinforce the learning of individual parameters. This approach is novel in the field since most of PK/PD modellers do not use prior information from other studies but fit exclusively clinical data of interest . Heterogeneity is then addressed by mixed effects modeling where parameters estimation is based on likelihood maximization for the entire population. In this case, assessment of algorithm's convergence and overfitting are controlled exclusively for population parameters determining the distributions of individual parameters. Consequently, mixed effects modeling derives individual parameter estimates as a by-product implying high probability of insufficient fitting quality for a significant number of subjects. Moreover, pre-assumptions on the parameter distributions could spoil individual fits as well. This limits the usefulness of these methods to develop individualized therapy predictions. In contrast, our approach maximizes individual fitting precision without making any pre-assumptions on the underlying parameter distributions. We controlled convergence of fitting algorithm and reported standard errors on an individual level. We believe that such an individualized control of goodness of fit is more appropriate for the purpose of individualized treatment management than classical mixed effects modelling.

It is of high practical importance to compare the predictive power of models of different complexity since more complex models usually have more parameters, and with it, are more prone to over-fitting. Indeed, numbers of parameters with assumed IIV differed between the models and was determined on the basis of an identifiability analysis. Since the mechanistic model uses external data to stabilize parameter estimation, more individual parameters could be estimated for it.

Our proposed comparison framework is based on the prediction performance of next-cycle toxicities using individual parameter sets derived from individual blood counts observed so far. This situation is close to clinical practice where physicians have to decide upon treatment continuation after completion of a chemotherapy cycle. We used real patient data for our comparison but needed to restrict the analysis to patients with a sufficient number of observations per cycle, which are not commonly available in clinical practice though.

Models are fitted to data aiming at improving the agreement with observed thrombocytopenia grades. We used this approach to account for the fact that the degree of thrombopoenia is typically considered for clinical 
decision making. In contrast, agreement of model and data for large platelet counts is of lesser clinical utility. To achieve this goal, we introduced a novel transformation of platelets counts which continuously interpolates between thrombocytopenia grades. This transformation is characterized by an increased importance of correctly fitting low thrombocytopenia degrees as described in Supporting information S.4. The same fitness function was used for calibrating both models.

For comparisons of the predictive performance of our models, we defined two scores DD and LDD. Both measured deviations of predicted and observed degrees of thrombocytopenia, but the latter only counts deviations of more than one degree which is supposed to be of high clinical relevance. DD depended less than LDD on the number of cycles used for model calibration. We also determined the Youden index for the prediction of severe grade 3-4 thrombopoenia. It revealed that the MM outperforms the SMM irrespective of the evaluation function (DD, LDD, Youden index). Of note, the MM calibrated with only one cycle typically outperformed the SMM calibrated with a higher number of cycles except for highly unbalanced situations (e.g. where the SMM is calibrated on five cycles). The difference between models is less pronounced, when using standard errors of platelet counts as goal function (see supplement Figure S.6.1 in Supporting information S.6). This can be explained by the fact that the majority of data points are from periodic oscillations which are easy to capture by both models. However, for clinical utility it is much more important that critically low values are correctly predicted explaining our choice of goal functions.

We showed that regression analysis of clinical factors can be used to some extent to derive individual parameter estimates, e.g. when no platelet dynamics are not available. However, the predictive performance of these parameters is inferior compared to those obtained after calibrating the models to platelet data.

\section{Conclusion}

We conclude that our mechanistic model has superior predictive power regarding next cycle thrombotoxicity compared to the semi-mechanistic model proposed by Henrich et al. Time series data of only one cycle are required to achieve sufficiently accurate predictions of next-cycle toxicity. Based on our model, we developed a tool intended to support clinical decision making regarding next-cycle management in dependence on the individual therapy response. A prototype can be found elsewhere: (https://www.health-atlas.de/shinypublic/apps/thrombopenia/). We plan to assess its clinical utility in the future.

\section{Acknowledgements}

This work was supported by following grants for scientific research: HaematoOPT, German Federal Ministry of Research and Education, 031A424.

\section{Conflicts of Interest}

There are no conflicts of interest to declare.

\section{Data Sharing}

We are not allowed to make the patient data freely available because this is not covered by the respective consents. 


\section{Figure Legends}

Figure 1. Schematic structure of the semi-mechanistic model of Henrich et al. and our comprehensive mechanistic model of thrombocytopoiesis compared in this work. We present major compartments as boxes and cell or TPO fluxes as black arrows. Actions are depicted as thick lines with arrows (stimulation) respectively orthogonal lines (suppression). Different colors correspond to different kinds of actions.

Figure 2. Distribution of degrees of thrombocytopenia observed at different treatment cycles (primary data set of 135 patients from NHL-B trial). Percentages of patients with thrombocytopenia degree 0 is continuously decreasing from cycle to cylce. Percentages of patients with degrees 3 or 4 increase continuously. In particular, degree 4 thrombocytopenias only occur from cycle 3 and increase up to $10.1 \%$ at cycle 6 .

Figure 3. Comparisons of model predictions and data for the mechanistic and semi-mechanistic model for four selected patients from the NHL-B trial with either nearly periodic behavior (patient \#69) or with strong cumulative platelet toxicity during the course of the therapy (patients \#814, \#1678, \#1261). Model performances are comparable for these cases. Compared to the semi-mechanistic model, the mechanistic model performs slightly inferior at the first cycle but better at later cycles 5 and 6 ).

Figure 4. Comparisons of model predictions and data for the mechanistic and the semi-mechanistic model for eight patients from NHL-B study with irregular platelet dynamics during the course of the therapy with (patients \#37, \#398, \#489) or without clear cumulative toxicity (patients\#14, \#15 and \#677, \#1017, \#1428). Both models fail to fit the degree of thrombocytopenia correctly. The mechanistic model performs better for patients \#14, \#15, \#398 and \#1017.

Figure 5. Comparison of the predictive powers of the mechanistic and the semi-mechanistic model. Models are either calibrated on a prescribed number of previous cycles (x-axis) or used parameter settings obtained from regression models of baseline covariates from the validation data set (no calibration cycles, yellow). We present the average of the absolute differences between predicted and observed degrees of thrombocytopenia for future cycles (DD characteristics, y-axis). Color-coding corresponds to the predicted cycle. The mechanistic model outperforms the semi-mechanistic model in all situations except for the parameters derived from the regression model showing similar predictive power. The mechanistic model calibrated on the first cycle even outperforms the semi-mechanistic model calibrated on a higher number of cycles.

Figure 6. Comparison of the predictive powers of the mechanistic and the semi-mechanistic model. Models are calibrated on a prescribed number of cycles (x-axis) or used parameter settings obtained from regression models of baseline covariates from the validation data set (no calibration cycles, yellow). We present the average of the absolute differences between predicted and observed degrees of thrombocytopenia for future cycles, but compared to Figure 5, only differences of more than one degree are counted (LDD characteristics, y-axis). Color-coding corresponds to the predicted cycle. The mechanistic model outperforms the semimechanistic model in all situations except for the parameters derived from the regression model showing similar predictive power. The mechanistic model calibrated on the first cycle even outperforms the semimechanistic model calibrated on a higher number of cycles for most of the cases. The advantage of the mechanistic model is more pronounced than in Figure 5.

Figure 7. Comparison of sensitivities regarding grade III/IV toxicity of the mechanistic and the semimechanistic model. Models are calibrated on a prescribed number of cycles (x-axis). The sensitivity of the mechanistic model is higher.

Figure 8. Comparison of Youden's J statistics regarding grade III/IV toxicity of the mechanistic and the semi-mechanistic model. Models are calibrated on a prescribed number of cycles (x-axis). Youden's J statistics are higher throughout indicating higher predictive power. 
Semi-mechanistic model (Friberg et al, 2002)

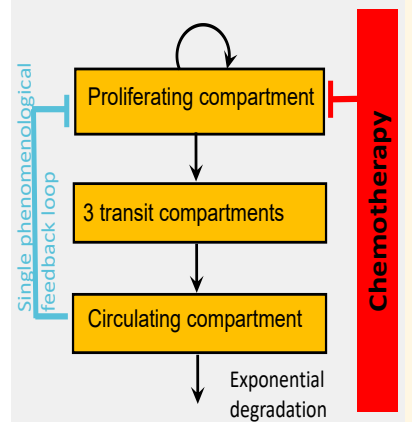

Parameters

- 3 estimated

- 3 parsimonies

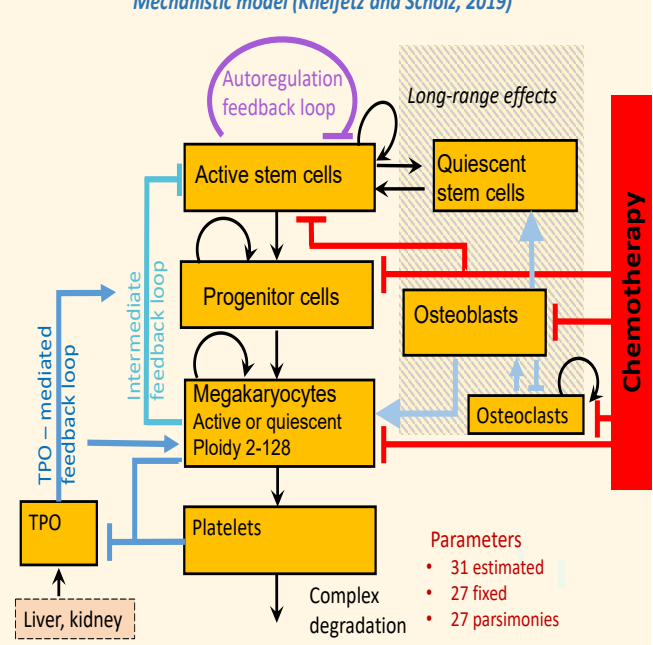




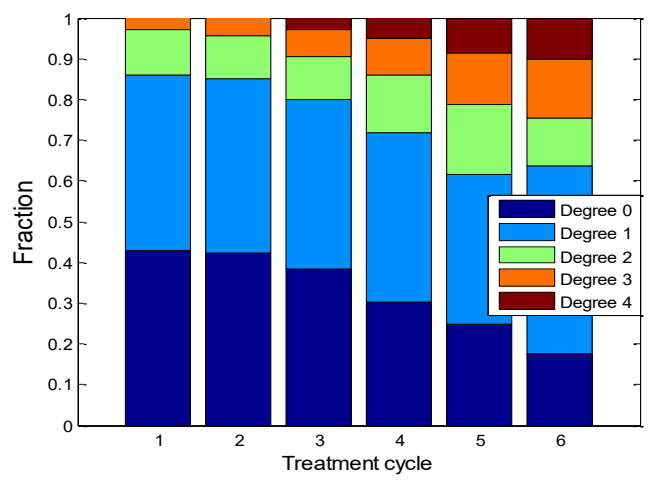



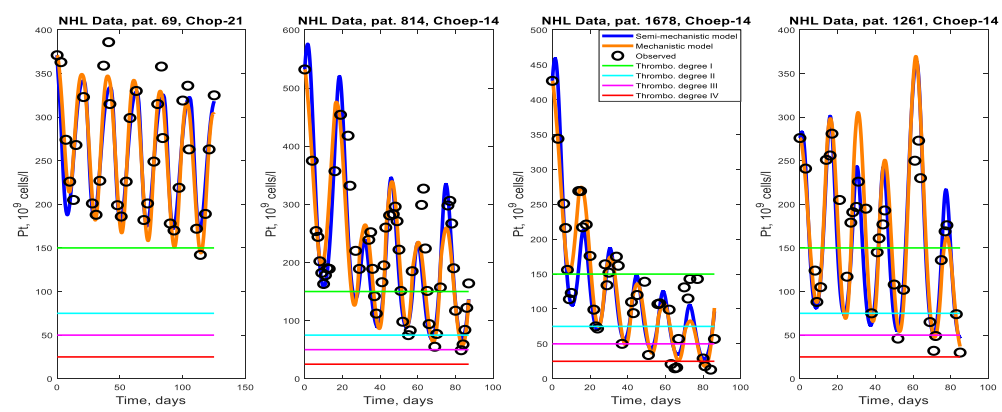

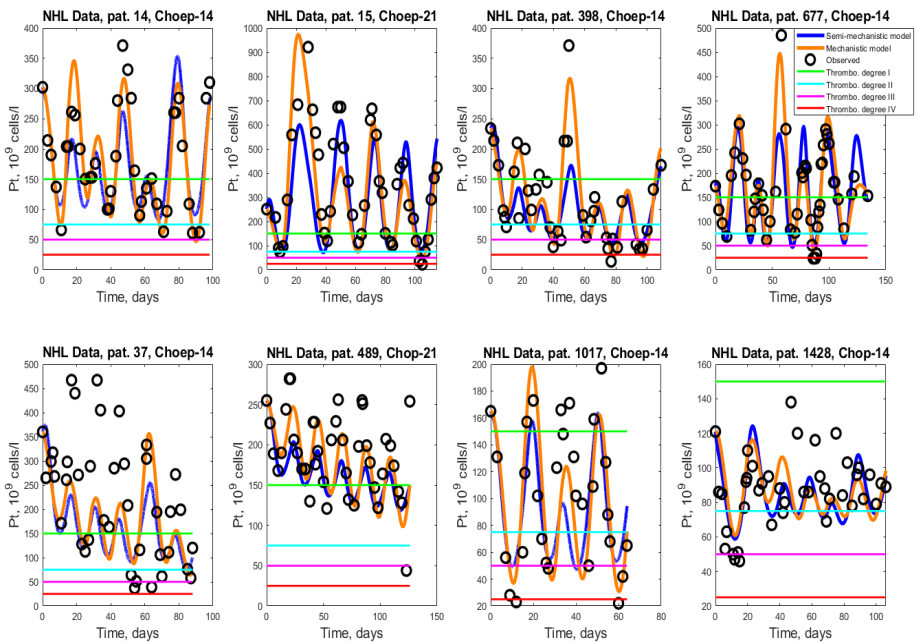

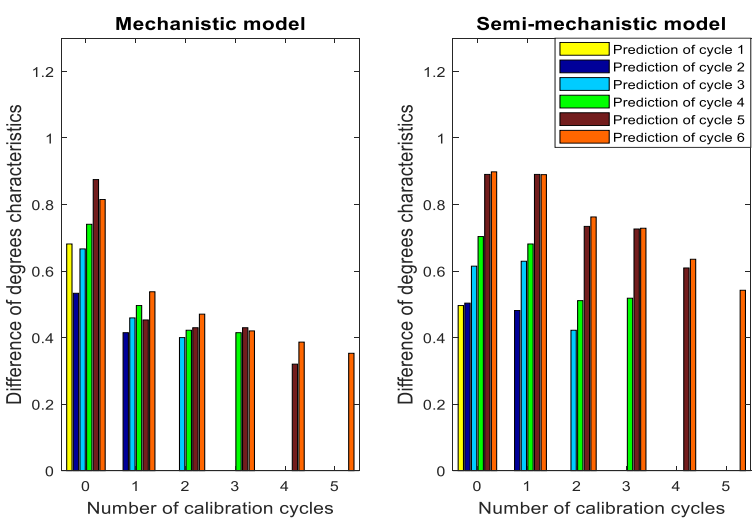

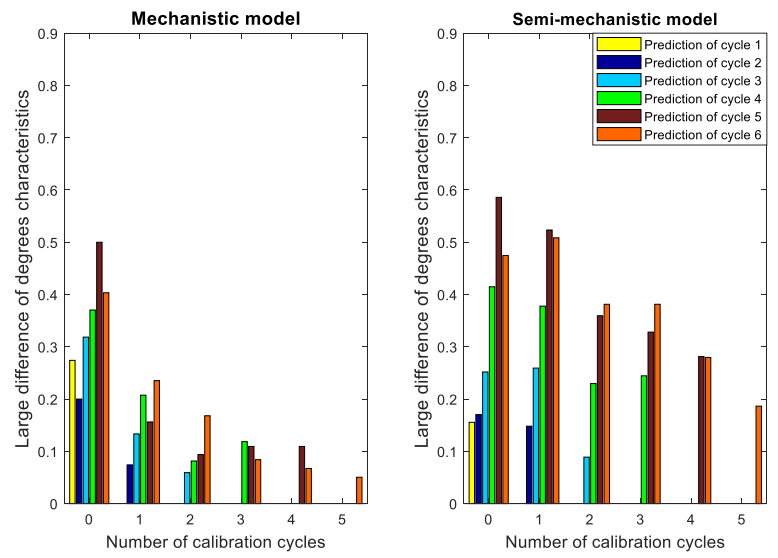

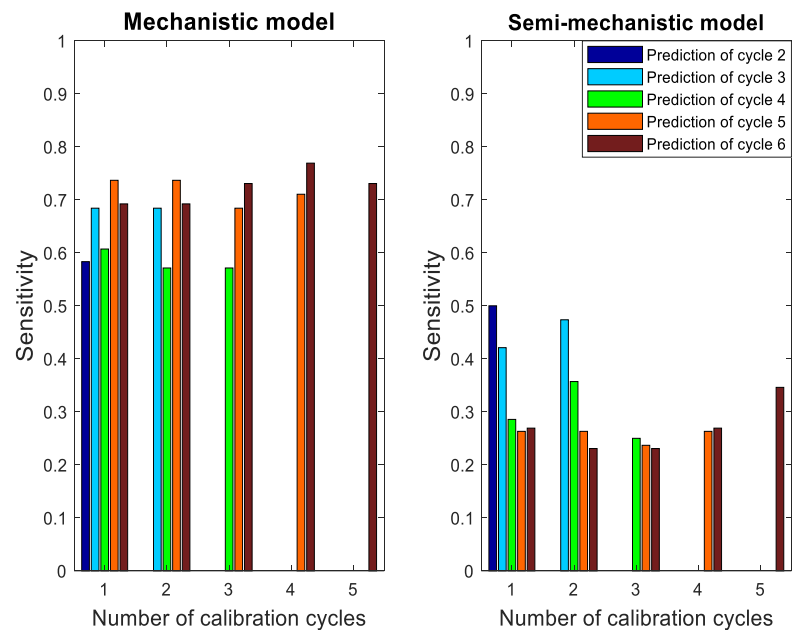

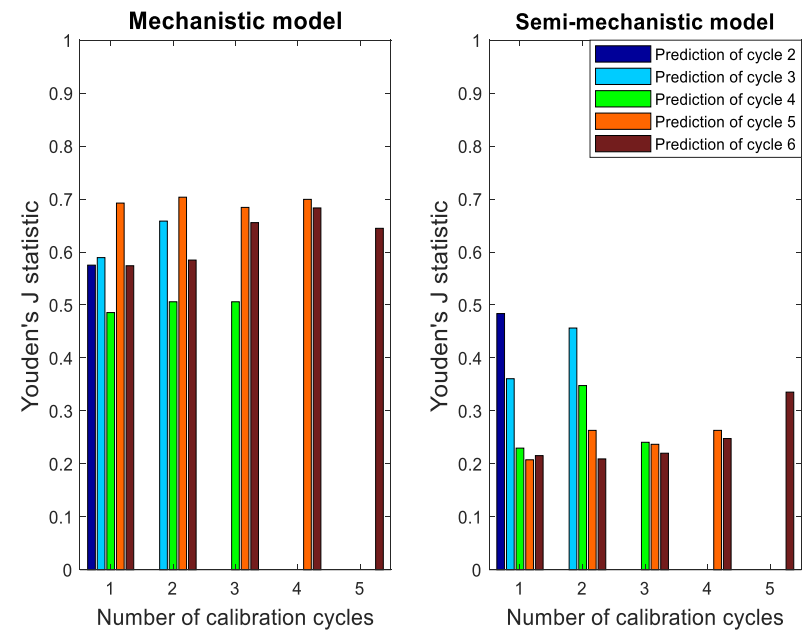